\title{
Stochastic Stability of Linear Time-Delay System with Markovian Jumping Parameters
}

\author{
K. BENJELLOUN and E. K. BOUKAS*,† \\ Mechanical Engineering Department, École Polytechnique de Montréal, \\ P.O. Box 6079, Station "centre-ville", Montréal, Québec, Canada, H3C \\ $3 A 7$
}

(Received 12 February 1996; Revised 30 April 1996)

This paper deals with the class of linear time-delay systems with Markovian jumping parameters (LTDSMJP). We mainly extend the stability results of the deterministic class of linear systems with time-delay to this class of systems. A delay-independent necessary condition and sufficient conditions for checking the stochastic stability are established. A sufficient condition is also given. Some numerical examples are provided to show the usefulness of the proposed theoretical results.

Keywords: stochastic stability; Markov process; linear systems with jumping parameters; time-delay

Classification Categories: 43E15

\section{INTRODUCTION}

This paper deals with stochastic stability of the class of linear time-delay systems with Markovian jumping parameters. The class of linear systems with Markovian jumping parameters (LSMJP) is a hybrid class of systems with two components in the vector state. The first component refers to the

\footnotetext{
*Corresponding author. E-mail: Boukas@anas.meca.polymtl.ca.

${ }^{\dagger}$ Research of this author was supported by the Natural Sciences and Engineering Research Council of Canada under grants OGP0036444.
} 
mode and the second one to the state. The mode is described by a continuous Markov process with finite state space. The state in each mode is represented by a differential equation.

This class of systems have received the attention of many researchers from both categories theoretical and practical. Many papers have been reported in the literature. Among these papers, we can quote Kats and Krasovskii [1], Sworder [2], Mariton and Bertrand [3], Ji and Chizeck [4], [5], [6], Kushner [7], Mariton [8], Feng et al [9], Boukas [10] and the references therein. In these cited papers the authors covered a large variety of problems such as stochastic stability, stochastic controllability, stochastic observability, optimal control, etc.

For the class of linear time-delay systems with Markovian jumping parameters, no work have been reported to the literature and the established results regarding the deterministic linear time-delay systems have not been extended yet to this class. For the deterministic linear time-delay systems there exists many papers in the literature. We quote Thowsen [11], Cheres [12], Ikeda [13], Lewis [14], Brierley [15], Mori [16] and the references therein.

The aim of this paper is to extend some results on the stability of the class of linear time-delay systems to our class of systems. We mainly establish a delay-independent sufficient condition to check the stochastic stability of LTDSMJP. A such condition is easy to check. A necessary condition which is easy to test is also established.

The paper is organized as follows: In section 2 , the stability problem is stated for the class of systems under consideration. In section 3, a sufficient condition for stochastic stability of this class is established. Other conditions, (necessary, sufficient), are also established. In section 4, some examples are presented to show the usefulness of the proposed theoretical results.

\section{PROBLEM STATEMENT}

In this paper, we consider the class of linear time-delay systems with Markovian jumping parameters where the dynamics is described by the following systems of differential equations: 


$$
\begin{aligned}
& \dot{\mathbf{x}}(t)=\mathbf{A}_{0}(\mathbf{r}(t)) \mathbf{x}(t)+\sum_{q=1}^{N} \mathbf{A}_{q}(\mathbf{r}(t)) \mathbf{x}\left(t-\tau_{q}\right) \\
& \mathbf{x}(t)=\Psi(t), t \in\left[t_{0}-\tau_{m}, t_{0}\right]
\end{aligned}
$$

where $t_{0}$ is the initial time (in the rest of the paper we will take $t_{0}=0$ ) and $\tau_{1}, \tau_{2}, \ldots, \tau_{N}$ are the system's constant delays and $\tau_{m}=\max _{q=1, \ldots, N}\left\{\left\{_{\tau q}\right\}\right.$, $\mathbf{x}(t) \in \mathbb{R}^{n}$ is the state vector at time $t, \Psi(t) \in \mathscr{C}\left(\left[-\tau_{m}, 0\right] \rightarrow \mathbb{R}^{n}\right)$ is a continuous vector-valued initial function, $\left(\mathscr{C}\left(\left[-\tau_{m}, 0\right] \rightarrow \mathbb{R}^{n}\right)\right.$ is the space of continuous functions mapping $\left[-\tau_{m}, 0\right]$ into $\left.\mathbb{R}^{n}\right)$ and $\mathbf{A}_{0}(\mathbf{r}(t)) \in \mathbb{R}^{n \times n}$ and $\mathbf{A}_{q}(\mathbf{r}(t)) \in \mathbb{R}^{n \times n}, q=1, \ldots, N$ are matrices with real values. These matrices are functions of the random parameter $\mathbf{r}(t)$.

The parameter $\mathbf{r}(t)$ in Eq. (1) represents a continuous-time discrete-state Markov process with values in a finite set $\mathscr{S}=\{1,2, \ldots, s\}$ with transition probability rate matrix $\Pi=\left[\pi_{i j}\right]_{i, j=1, s}$. The transition probability from mode $i$ to mode $j$ is given by:

$$
P\{\mathbf{r}(t+\Delta)=j \mid \mathbf{r}(t)=i\}= \begin{cases}\pi_{i j} \Delta+o(\Delta) & i \neq j \\ 1+\pi_{i i} \Delta+o(\Delta) & i=j\end{cases}
$$

where $\Delta>0$ and $\lim _{\Delta \rightarrow 0} \frac{o(\Delta)}{\Delta}$.

In this Eq. (3), $\left(\pi_{i j} \geq 0\right)$ is the transition probability rate from mode $i$ to mode $j(i \neq j)$ and

$$
\sum_{j=1 j \neq}^{s} \pi_{i j}=-\pi_{i i}=\pi_{i} \text { for } i=1, \ldots, s
$$

\section{Remarks 2.1}

- The system described by Eqs. (1)-(3) is a hybrid system with state vector $(\mathbf{r}(t), \mathbf{x}(t))$;

- For each mode $i$, the system described by Eqs. (1)-(3) is linear in $\mathbf{x}(t)$, $\mathbf{x}\left(t-\tau_{1}\right), \ldots, \mathbf{x}\left(t-\tau_{q}\right)$.

To simplify the study of the class of systems under consideration, we will assume that the system delays are constant and not dependent on the stochastic process but depend on the system structure. Let the initial val- 
ues $\{\Psi(t)\}_{-\tau_{m \leq t \leq 0}}$ and $\{\rho(t)=\mathbf{r}(t)\}_{-\tau_{m \leq t \leq 0}}$ be independent random functions and fixed. Furthermore, we assume that the system has the same dimension at each mode and the Markov process is irreducible.

The question we address in this paper is how to check the stability of this class of systems. In the rest of this paper, we will establish the necessary or sufficient condition for the stochastic stability, and some examples are given to illustrate our results.

When the mode of the system is $i$, i.e. $\mathbf{r}(t)=i$, we will use the notation $\mathbf{A}_{q i}$ to represent the matrix $\mathbf{A}_{q}(\mathbf{r}(t))$ with $q=0,1, \ldots, N .\|\mathbf{x}\|=\left(x_{1}^{2}+\right.$ $\left.x_{2}^{2}+\cdots+x_{n}^{2}\right)^{1 / 2}$ (where $x_{i}$, for $i=1, \ldots, n$, denotes the $i$ th element of $\left.\mathbf{x}(t)\right)$ represents the Euclidean norm of vector $\mathbf{x}$. The induced Euclidean norm of matrix $\|\mathbf{M}\|$ is given by $\|\mathbf{M}\|=\left[\lambda_{\max }\left(\mathbf{M}^{\prime} \mathbf{M}\right)\right]^{1 / 2}$, where $\mathbf{M}^{\prime}$ denotes the transpose of matrix $\mathbf{M}$ and $\lambda_{\min }(\mathbf{M})$ and $\lambda_{\max }(\mathbf{M})$ denote respectively the minimum eigenvalue and the maximum eigenvalue of matrix (M). $\mathbf{M}>0$ means that the matrix $\mathbf{M}$ is positive-definite and $\mathbf{M}^{1 / 2}$ is the matrix square root.

\section{STOCHASTIC STABILITY OF LTDSMJP}

The concept of stochastic stability for linear and nonlinear systems was used by many authors. Among these authors, we quote the research of Kats and Krasovskii [1], Mariton [8], Ji and Chizeck [6] and the references therein. In this paper, we will use the following definition:

Definition Let $E\{$.$\} be the expectation, \mathbf{x}_{0}=\mathbf{x}(0)$ be the initial state of the system and $\mathbf{x}(t, \Psi, \rho)$ be the corresponding solution of system (1)-(3) at time $t$ when the initial conditions are respectively $\Psi$ and $\rho$. The system (1)-(3) is stochastically stable if for all finite $\Psi(\mathrm{t}) \in \mathscr{C}\left(\left[-\tau_{m}, 0\right] \rightarrow \mathbb{R}^{n}\right)$, and $\rho\left(\left[-\tau_{m}, 0\right] \rightarrow \mathscr{Y}\right.$, there exists a constant $\tilde{c}$ such that

$$
\lim _{T \rightarrow \infty} E\left\{\int_{0}^{T} \mathbf{x}^{\prime}\left(t, \Psi, \mathbf{r}_{0}\right) \mathbf{x}(t, \Psi, \rho) d t \mid \Psi \mathbf{r}_{0}\right\} \leq \tilde{c}\left\|\mathbf{x}_{0}\right\|^{2}
$$

is satisfied.

The following assumption establishes a relation between the system state at time $t$ and the one at time $t+\eta, \eta \in\left[-\tau_{m}, 0\right]$. 
Assumption A (Kolmanovskii [17]) For all $\eta \in\left[-\tau_{m}, 0\right]$, there exists a scalar $h>1$ such that

$$
\|\mathbf{x}(t+\eta)\| \leq h\|\mathbf{x}(t)\|
$$

where $\mathbf{x}(t)$ is the state of system at time $t$.

Based on the results of Ji and Chizeck [6] for the class of linear systems with Markovian jumps and the ones of Gregory and Nazaroff [18] for the class of linear systems with delay, we can establish the following delayindependent sufficient condition for stochastic stability:

THEOREM 1 The system (1)-(3) is stochastically stable if for any given positive-definite symmetric matrix $\mathbf{Q}_{\mathrm{i}}, \forall \mathrm{i} \in \mathscr{S}$ and a constant positivedefinite symmetric matrix $\mathbf{Q}$, the unique set of solutions, $\mathbf{P}_{\mathrm{i}}, \forall \mathrm{i} \in \mathscr{Y}$, of the coupled system:

$$
\mathbf{A}_{0 i}^{\prime} \mathbf{P}_{i}+\mathbf{P}_{i} \mathbf{A}_{0 i}+\mathbf{P}_{i}\left[\sum_{q=1}^{N} \mathbf{A}_{q i} \mathbf{Q}^{-1} \mathbf{A}_{q i}^{\prime}\right] \mathbf{P}_{i}+\sum_{j=1}^{s} \pi_{i j} \mathbf{P}_{j}+N \mathbf{Q}=-\mathbf{Q}_{i}
$$

is positive-definite symmetric.

Proof Let the mode at time $t$ be $i$, i.e. $\mathbf{r}(t)=i \in \mathscr{Y}$; then Eq. (1) becomes:

$$
\dot{\mathbf{x}}(t)=\mathbf{A}_{0 i} \mathbf{x}(t)+\sum_{q=1}^{N} \mathbf{A}_{q i} \mathbf{x}\left(t-\tau_{q}\right)
$$

Based on the results of Kushner [7] and Gregory and Nazaroff [18], we can choose the following stochastic Lyapunov functional $V():. \mathbb{R}^{n} \times \mathscr{S} \rightarrow$ $\mathbf{R}_{+}$:

$$
\begin{aligned}
V(\mathbf{x}(t), \mathbf{r}(t)=i) & =V(\mathbf{x}, i) \\
& =\mathbf{x}^{\prime}(t) \mathbf{P}_{\mathbf{i}} \mathbf{x}(t)+\sum_{q=1}^{N} \int_{t-\tau_{q}}^{t} \mathbf{x}^{\prime}(\eta) \mathbf{Q} \mathbf{x}(\eta) d \eta
\end{aligned}
$$

The infinitesimal operator $\tilde{\mathscr{A}}$ of the random process $\{(\mathbf{x}(t), \mathbf{r}(t)), t \geq 0\}$ is given by: 


$$
\begin{aligned}
\tilde{\mathscr{A}} V(\mathbf{x}(t), \mathbf{r}(t))= & \lim _{\Delta \rightarrow 0} \frac{1}{\Delta}[E\{V(\mathbf{x}(t+\Delta), \mathbf{r}(t+\Delta) \mid \mathbf{x}(t), \mathbf{r}(t)=i\} \\
& -V(\mathbf{x}(t), \mathbf{r}(t)=i)] \\
= & \mathbf{x}^{\prime}(t)\left[\mathbf{A}_{0 i}^{\prime} \mathbf{P}_{i}+\mathbf{P}_{i} \mathbf{A}_{0 i}+\sum_{j=1}^{s} \pi_{i j} \mathbf{P}_{j}+N \mathbf{Q}\right] \mathbf{x}(t) \\
& +2 \sum_{q=1}^{N} \mathbf{x}^{\prime}(t) \mathbf{P}_{i} \mathbf{A}_{q i} \mathbf{x}\left(t-\tau_{q}\right)-\sum_{q=1}^{N} \mathbf{x}^{\prime}\left(t-\tau_{q}\right) \mathbf{Q} \mathbf{x}\left(t-\tau_{q}\right)
\end{aligned}
$$

Based on Eq. (6), we obtain:

$$
\begin{aligned}
& \tilde{A} V(\mathbf{x}, i)=-\mathbf{x}^{\prime}(t) \mathbf{Q}_{i} \mathbf{x}(t)-\mathbf{x}^{\prime}(t) \mathbf{P}_{i}\left[\sum_{q=1}^{N} \mathbf{A}_{q i} \mathbf{Q}^{-1} \mathbf{A}_{q i}^{\prime}\right] \mathbf{P}_{i} \mathbf{x}(t) \\
&+2 \sum_{q=1}^{N} \mathbf{x}^{\prime}(t) \mathbf{P}_{i} \mathbf{A}_{q i} \mathbf{x}\left(t-\tau_{q}\right)- \\
& \sum_{q=1}^{N} \mathbf{x}^{\prime}\left(t-\tau_{q}\right) \mathbf{Q} \mathbf{x} \\
&\left(t-\tau_{q}\right) \\
&=- \sum_{q=1}^{N}\left(( \mathbf { Q } ^ { 1 / 2 } \mathbf { x } ( t - \tau _ { q } ) - \mathbf { Q } ^ { 1 / 2 } \mathbf { A } _ { q i } ^ { \prime } \mathbf { P } _ { i } \mathbf { x } ( t ) ) ^ { \prime } \left(\left(\mathbf{Q}^{1 / 2} \mathbf{x}\left(t-\tau_{q}\right)\right.\right.\right. \\
&\left.-\mathbf{Q}^{-1 / 2} \mathbf{A}_{q i}^{\prime} \mathbf{P}_{i} \mathbf{x}(t)\right)-\mathbf{x}^{\prime}(t) \mathbf{Q}_{i} \mathbf{x}(t)
\end{aligned}
$$

Notice that $\left(Q^{1 / 2} \mathbf{x}\left(t-\tau_{q}\right)-\mathbf{Q}^{-1 / 2} \mathbf{A}_{q i}^{\prime} \mathbf{P}_{i} \mathbf{x}(t)\right)^{\prime}\left(\mathbf{Q}^{1 / 2} \mathbf{x}\left(t-\tau_{q}\right)-\mathbf{Q}^{-1 / 2} \mathbf{A}_{q i}^{\prime} \mathbf{P}_{i} \mathbf{x}\right.$ $(t))$ is a positive number. Then we have:

$$
\tilde{A} V(\mathbf{x}, i) \leq-\mathbf{x}^{\prime}(t) \mathbf{Q}_{i} \mathbf{x}(t)
$$

On the other side, for $\mathbf{x} \neq 0$ and for each mode $i$, we have:

$$
V(\mathbf{x}, i) \geq \mathbf{x}^{\prime} \mathbf{P}_{i} \mathbf{x}>0
$$

Thus, 


$$
\frac{\tilde{A} V(\mathbf{x}, i)}{V(\mathbf{x}, i)} \leq-\frac{\mathbf{x}^{\prime} \mathbf{Q}_{i} \mathbf{x}}{\mathbf{x}^{\prime} \mathbf{P}_{i} \mathbf{x}} \text { for } \mathbf{x} \neq 0
$$

Using the fact that $\mathbf{Q}_{i}>0$ and $\mathbf{P}_{i}>0$, we can write

$$
\frac{\tilde{A} V(\mathbf{x}, i)}{V(\mathbf{x}, i)} \leq-\beta:=-\min _{i \in \mathscr{Y}}\left\{\frac{\lambda_{\min }\left(\mathbf{Q}_{i}\right)}{\boldsymbol{\lambda}_{\max }\left(\mathbf{P}_{i}\right)}\right\}
$$

Using now Dynkin's formula [7], the Fubini's theorem and the Gronwall-Bellman lemma [19], we get for each $i \in \mathscr{Y}$ :

$$
E\left\{V(\mathbf{x}(t), \mathbf{r}(t)=i) \mid \mathbf{x}_{0}, \mathbf{r}_{0}=i\right\} \leq e^{-\beta t} V\left(\mathbf{x}_{0}, i\right)
$$

Thus,

$$
\begin{aligned}
& E\left\{V(\mathbf{x}, i) \mid \mathbf{x}_{0}, \mathbf{r}_{0}=i\right\} \\
& =E\left\{\mathbf{x}^{\prime}(t) \mathbf{P}_{i} \mathbf{x}(t)+\sum_{q=1}^{N} \int_{t-\tau_{q}}^{t} \mathbf{x}^{\prime}(\eta) \mathbf{Q} \mathbf{x}(\eta) d \eta \mid \mathbf{x}_{0}, \mathbf{r}_{0}=i\right\} \\
& =E\left\{\mathbf{x}^{\prime}(t) \mathbf{P}_{i} \mathbf{x}(t) \mid \mathbf{x}_{0}, \mathbf{r}_{0}=i\right\}+E\left\{\sum_{q=1}^{N} \int_{t-\tau_{q}}^{t} \mathbf{x}^{\prime}(\eta) \mathbf{Q x}(\eta) d \eta \mid \mathbf{x}_{0}, \mathbf{r}_{0}=i\right\} \\
& \quad \leq e^{-\beta t} V\left(\mathbf{x}_{0}, i\right)
\end{aligned}
$$

Notice that $\mathrm{E}\left\{\sum_{q=1}^{\mathrm{N}} \int_{t-\tau_{q}}^{t} \mathbf{x}^{\prime}(\eta) \mathbf{Q x}(\eta) d \eta \mid \mathbf{x}_{0}, \mathbf{r}_{0}=i\right\}$ is a positive number, then

$$
E\left\{\mathbf{x}^{\prime}(t) \mathbf{P}_{i} \mathbf{x}(t) \mid \mathbf{x}_{0}, \mathbf{r}_{0}=i\right\} \leq e^{-\beta t} V\left(\mathbf{x}_{0}, i\right)
$$

Let $\lambda_{\mathrm{m}}:=\min _{\mathrm{i} \in \mathcal{S}}\left\{\lambda_{\min }\left(\mathbf{P}_{i}\right)\right\}, \lambda_{M}:=\max _{i \in \mathscr{S}}\left\{\lambda_{\max }\left(\mathbf{P}_{i}\right)\right\}$ and using once more Fubini's theorem we have: 


$$
\begin{aligned}
& E\left\{\int_{0}^{T} \mathbf{x}^{\prime}(t, \psi, \rho) \mathbf{P}_{i} \mathbf{x}(t, \psi, \rho) d t \mid \mathbf{x}_{0}, \mathbf{r}_{0}=i\right\} \leq\left(\int_{0}^{T} e^{-\beta t} d t\right) V\left(\mathbf{x}_{0}, i\right) \\
& \lambda \mathrm{E}\left\{\int_{0}^{\mathrm{T}} \mathbf{x}^{\prime}(t, \psi, \rho) \mathbf{x}(t, \psi, \rho) d t \mid \psi, \mathbf{r}_{0}=i\right\} \leq-\frac{1}{\beta}\left[e^{-\beta T}-1\right] V\left(\mathbf{x}_{0}, i\right)
\end{aligned}
$$

Let $\tau=\sum_{q=1}^{\mathrm{N}} \tau_{q}$, using the assumption $\mathbf{A}$ and taking the limit as $T \rightarrow \infty$, we obtain:

$$
\begin{aligned}
\lim _{T \rightarrow \infty} E\left\{\int_{0}^{T} \mathbf{x}^{\prime}(t, \psi, \rho) \mathbf{x}(t, \psi, \rho) d t \mid \mathbf{x}_{0}, \mathbf{r}_{0}=i\right\} & \leq \frac{1}{\lambda_{m} \beta} \mathbf{x}_{o}^{\prime}\left(\mathbf{P}_{i}+\tau \mathbf{h}^{2} \boldsymbol{\lambda}_{\max }(\mathbf{Q}) \mathbf{I}\right) \mathbf{x}_{0} \\
& \leq \tilde{c}\left\|\mathbf{x}_{0}\right\|^{2}
\end{aligned}
$$

where

$$
\tilde{c}:=\frac{1}{\lambda_{m} \beta}\left[\lambda_{\max }\left(\mathbf{P}_{i}\right)+\mathbf{h}^{\mathbf{2}} \boldsymbol{\tau} \boldsymbol{\lambda}_{\max }(\mathbf{Q})\right]
$$

This completes the proof of the Theorem 1 .

Remarks 3.1

- If the system has only a single mode, then condition (6) is reduced to the condition of the deterministic stability of linear time-delay system.

- When the time delay is equal to 0 , we obtain the result established by Ji and Chizeck [6]. The Eq. (6) can be rewritten as:

$$
\left(\mathbf{A}_{0 i}+\sum_{q=1}^{N} \mathbf{A}_{q i}\right) \mathbf{P}_{i}+\mathbf{P}_{i}\left(\mathbf{A}_{0 i}+\sum_{q=1}^{N} \mathbf{A}_{q i}\right)+\sum_{j=1}^{s} q_{i j} \mathbf{P}_{\mathrm{j}}=-\mathbf{Q}_{i}
$$

- Notice that the proposed result doesn't depend on the delay of the system.

- Notice that the condition of Theorem 1 given by Eq. (6) is similar to the following conditions:

$$
\left\{\begin{array}{l}
\mathbf{A}_{0 \mathrm{i}}^{\prime} \mathbf{P}_{i}+\mathbf{P}_{i} \mathbf{A}_{0 \mathrm{i}}+\sum_{j=1}^{s} \pi_{i j} \mathbf{P}_{j}+-N \mathbf{Q}-\mathbf{Q}_{i} \\
\mathbf{Q}_{i}-\mathbf{P}_{i}\left[\sum_{q=1}^{N} \mathbf{A}_{q i} \mathbf{Q}^{-1} \mathbf{A}_{q i}^{\prime}\right] \mathbf{P}_{i}>\mathbf{0}
\end{array}\right.
$$

The first equation of the system (17) gives the condition of the stochastic stability of linear system with Markovian jumping parameters (LSMJP) given by (1)-(3) when $\mathbf{A}_{\boldsymbol{q} i}=\mathbf{0}$ for $q=1, \ldots, N$ and $i=1, \ldots, s$. 
The following corollary provides a necessary condition for stochastic stability which easier to test.

Corollary 1 If the system described by Eqs. (1)-(3) is stable in the stochastic sense, for each mode $\mathrm{i} \in \mathcal{Y}$, then the matrices $\left(\mathbf{A}_{0 i}+\right.$ $\left.\sum_{q i}^{N} \mathbf{A}_{q i}-1 / 2 \pi_{i} \mathbf{I}\right)$ and $\left(\mathbf{A}_{0 i}-1 / 2 \pi_{i} \mathbf{I}\right)$ have all its eigenvalues in the left half plane i.e. stable.

Proof Suppose that the system described by the Eqs. (1)-(3) is stochastically stable, i.e. for any given matrices $\mathbf{Q}_{i}, \forall i \in \mathscr{Y}$ and $\mathbf{Q}$, there exists $s$ unique matrices $\mathbf{P}_{i}$ satisfying the condition (6) (resp. (17)). Therefore, the systems

$$
\begin{aligned}
& \dot{\mathbf{x}}(t)=\mathbf{A}_{i} \mathbf{x}(t) \quad t \geq t_{0} \\
& \dot{\mathbf{x}}(t)=\left[\mathbf{A}_{i}+\sum_{q=1}^{N} \mathbf{A}_{q i}\right] \mathbf{x}(t) \quad t \geq t_{0}
\end{aligned}
$$

are stochastically stable. Using the corollary 1 , established by $\mathrm{Ji}$ an Chizeck [6], we conclude, that for each $i \in \mathscr{Y}$ the matrices $\mathbf{A}_{i}+\sum_{q=1}^{N} \mathbf{A}_{q i}-1 / 2 \pi_{\mathbf{i}} \mathbf{I}$ and $\mathbf{A}_{i}-\mathbf{1} / \mathbf{2} \boldsymbol{\pi}_{i} \mathbf{I}$ are stable.

Notice that the stability of the matrix $\mathbf{A}_{i}+\sum_{q=\mathbf{1}}^{N} \mathbf{A}_{q i}-1 / 2 \pi_{\mathbf{i}} \mathbf{I}$ or $\mathbf{A}_{i}-1 / 2 \pi_{i} \mathbf{I}$ for each mode $i \in \mathscr{Y}$ is not sufficient for the stochastic stability of (1)-(3), this is shown in example 4.2 of section 4.

The following corollary gives a delay-independent sufficient condition for stochastic stability of LTDSMJP which represents an easy test.

Corollary 2 Let $\mathbf{Q}_{\mathbf{i}} \in \mathbb{R}^{\mathrm{n} \times \mathrm{n}}$ be a given positive-definite symmetric matrix such that the system of $s$ coupled equations

$$
\mathbf{A}_{0 i}^{\prime} \mathbf{P}_{i}+\mathbf{P}_{i} \mathbf{A}_{0 \mathbf{i}}+\sum_{j=1}^{s} \pi_{i j} \mathbf{P}_{j}=-N \mathbf{Q}-\mathbf{Q}_{i}
$$

has a unique solution set of positive-definite symmetric matrix $\mathbf{P}_{i}$. Then the system (1)-(3) is stochastically stable if the following condition

is satisfied

$$
\sum_{q=1}^{N}\left\|\mathbf{A}_{q i}\right\|^{2}<v_{i}:=\frac{\lambda_{\min }\left(\mathbf{Q}_{i}\right) \lambda_{\min }(\mathbf{Q})}{\left(\lambda_{\max }\left(\mathbf{P}_{i}\right)\right)^{2}}, \quad \forall \mathbf{i} \in \mathscr{Y}
$$


Proof Take the stochastic Lyapunov functional given by Eq. (8) where $\mathbf{P}_{i}$ is symmetric positive-definite solution of system (18). Consider now, the weak infinitesimal operator $\tilde{\mathscr{A}}$ of the stochastic process $\{(\mathbf{x}(t), \mathbf{r}(\mathrm{t})), t \geq$ $0\}$ :

$$
\begin{gathered}
\tilde{\mathscr{A} V}(\mathbf{x}(t), \mathbf{r}(t)=i)=\mathbf{x}^{\prime}(t)\left[\mathbf{A}_{0}^{\prime} i \mathbf{P}_{i}+\mathbf{P}_{i} \mathbf{A}_{0 i}+\sum_{j=i}^{s} \pi_{i j} \mathbf{P}_{j}+N \mathbf{Q}\right] \mathbf{x}(t) \\
+2 \mathbf{x}^{\prime}(t) \mathbf{P}_{i} \sum_{q=1}^{N} \mathbf{A}_{q i} x\left(t-\tau_{q}\right)-N \mathbf{x}^{\prime}(t-\tau) \mathbf{Q} \mathbf{x}\left(t-\tau_{q}\right)
\end{gathered}
$$

Based on the Eq. (18) we have

$$
\begin{aligned}
\tilde{A} V(\mathbf{x}, i)= & -\mathbf{x}^{\prime}(t)\left(\mathbf{Q}_{i}-\mathbf{P}_{i} \sum_{q=1}^{N} \mathbf{A}_{q i} Q^{-1} \mathbf{A}_{q i}^{\prime} \mathbf{P}_{i}\right) \mathbf{x}(t) \\
& -\mathbf{x}^{\prime}(t) \mathbf{P}_{i} \sum_{q=1}^{N} \mathbf{A}_{q i} Q^{-1} \mathbf{A}_{q i}^{\prime} \mathbf{P}_{i} \mathbf{x}(t) \\
& +2 \mathbf{x}^{\prime}(t) \mathbf{P}_{i} \sum_{q=1}^{N} \mathbf{A}_{q i} x\left(t-\tau_{q}\right)-\sum_{q=1}^{N} \mathbf{x}^{\prime}\left(t-\tau_{q}\right) \mathbf{Q} \mathbf{x}\left(t-\tau_{q}\right) \\
= & -\sum_{q=1}^{N}\left(\mathbf{Q}^{1 / 2} \mathbf{x}\left(t-\tau_{q}\right)-\mathbf{Q}^{-1 / 2} \mathbf{A}_{1 i}^{\prime} \mathbf{P}_{i} \mathbf{x}(t)\right)^{\prime}\left(\mathbf{Q}^{1 / 2} \mathbf{x}\left(t-\tau_{q}\right)\right. \\
& \left.-\mathbf{Q}^{-1 / 2} \mathbf{A}_{1 i}^{\prime} \mathbf{P}_{i} \mathbf{x}(t)\right)-\mathbf{x}^{\prime}(t)\left(\mathbf{Q}_{i}-\mathbf{P}_{i} \sum_{q=1}^{N} \mathbf{A}_{q i} \mathbf{Q}^{-1} \mathbf{A}_{q i}^{\prime} \mathbf{P}_{i}\right) \mathbf{x}(t) \\
\leq & -\left[\lambda_{\min }\left(\mathbf{Q}_{i}\right)-\frac{1}{\lambda_{\min }(\mathbf{Q})}\left(\lambda_{\max }\left(\mathbf{P}_{i}\right)\right)^{2} \sum_{q=1}^{N}\left\|\mathbf{A}_{q i}\right\|^{2}\right]\|\mathbf{x}(t)\|^{2} \\
\leq & -\beta_{i}\|\mathbf{x}(t)\|^{2}
\end{aligned}
$$

where $\beta_{i}:=\boldsymbol{\lambda}_{\min }\left(\mathbf{Q}_{i}\right)-\frac{1}{\lambda_{\min }(\mathbf{Q})}\left(\lambda_{\max }\left(\mathbf{P}_{i}\right)\right)^{2} \Sigma_{q=1}^{N}\left\|\mathbf{A}_{q i}\right\|^{2}>0$.

The end of the proof is similar to the one presented for the proof of Theorem 1.

Remark 3.2 It can be seen from Theorem 1 that our result consists of solving a coupled Riccati equations type to check the stochastic stability 
of (1)-(3), which can be solved by the numerical algorithm of AbouKandil et al. [21].

\section{EXAMPLES}

In this section, we will present some examples to illustrate the usefulness of the proposed theoretical results. Also we will try to justify the features we point out in the previous remarks.

Example 4.1 Let us consider a system with two modes and continuous state $\mathbf{x}(t) \in \mathbb{R}^{2}$. Let the dynamics in each mode bedescribed as follows:

mode 1: $\quad \mathbf{A}_{01}=\left[\begin{array}{cc}0.5 & -1 \\ 0 & -3\end{array}\right], \quad \mathbf{A}_{11}=\left[\begin{array}{cc}0.5 & -0.2 \\ 0.2 & 0.3\end{array}\right]$

mode 2: $\quad \mathbf{A}_{02}=\left[\begin{array}{cc}-5 & 1 \\ 1 & 0.2\end{array}\right], \quad \mathbf{A}_{12}=\left[\begin{array}{rr}-0.3 & 0.5 \\ 0.4 & -0.5\end{array}\right]$

Let us also assume that the delay is the same in the two modes i.e. $\tau_{1}=$ $\tau_{2}=1 s$ and the initial conditions are $\psi(t)_{-\tau \leq 0}=[1,1]^{\prime}$. Finally let the generator matrix $\Pi$ of the stochastic process $\mathbf{r}(t)$ be:

$$
\Pi=\left[\begin{array}{rr}
-7 & 7 \\
6 & -6
\end{array}\right]
$$

and $\rho$ such that $\rho(t)_{-\tau \leq 0}=1$.

Let us choose for computation the following matrices $\mathbf{Q}_{1}=\mathbf{Q}_{2}=\mathbf{Q}=$ $\mathbf{I}_{2}$ where $\mathbf{I}_{2}$ denotes the $2 \times 2$ identity matrix. The solution of the corresponding coupled Riccati equations using the numerical algorithm given in [21] gives two symmetric and positive-definite matrices:

$$
P_{1}=\left[\begin{array}{ll}
1.0206 & 0.1123 \\
0.1123 & 1.2255
\end{array}\right], \quad P_{2}=\left[\begin{array}{ll}
0.5444 & 0.2837 \\
0.2837 & 1.9923
\end{array}\right]
$$

Based on Theorem 1, the system is then stochastically stable. A program written in Matlab was developed to simulate the evolution of the consid- 
ered system. The results of the simulation are shown in Figure 1, where the curves of the variables $x_{1}(t), x_{2}(t)$ and $r(t)$ versus time are plotted.

Remark Notice that the system in each mode is unstable. Therefore we conclude, that the stability in each mode is not necessary for the stochastic stability of the system.

The solution of the coupled Lyapunov equations (Eq. (18)) gives two positive-definite symmetric matrices:

$$
\mathbf{P}_{1}=\left[\begin{array}{ll}
0.9048 & 0.0553 \\
0.0553 & 0.8909
\end{array}\right], \quad \mathbf{P}_{2}=\left[\begin{array}{ll}
0.4898 & 0.2043 \\
0.2043 & 1.3847
\end{array}\right]
$$

Using condition (19), we obtain

$$
\left\|\mathbf{A}_{11}\right\|^{2}=0.3<v_{1}=1.1
$$
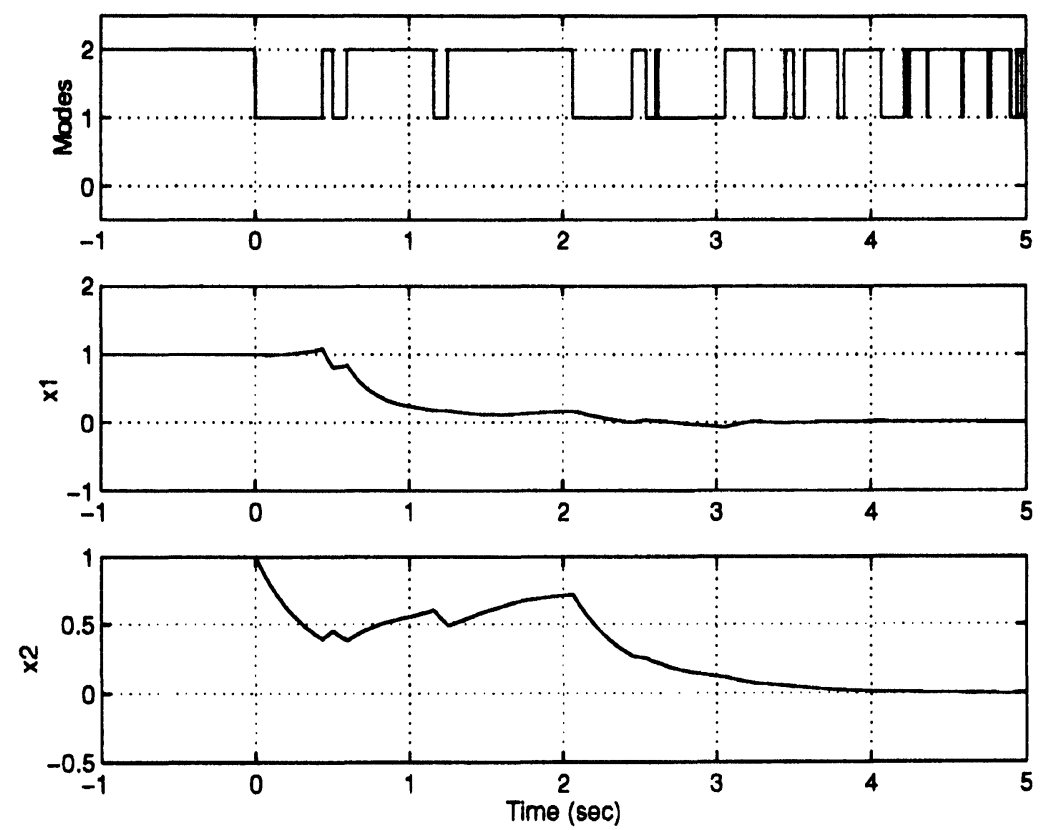

FIGURE 1 Evolution of modes and states versus time. 


$$
\left\|\mathbf{A}_{12}\right\|^{2}=0.75>v_{2}=0.5
$$

The condition of corollary 2 is not satisfied and consequently we can not conclude that the system under study is unstable, since the condition of this corollary is only sufficient.

EXAMPLE 4.2 Let us consider a dynamic system with continuous state in IRsup2 and two modes. Let the matrices in each mode be as follows:

mode 1: $\quad \mathbf{A}_{01}=\left[\begin{array}{ll}3 & -1 \\ 0 & -3\end{array}\right], \quad \mathbf{A}_{11}=\left[\begin{array}{rr}-4 & 1 \\ 0 & 1\end{array}\right]$

mode 2: $\quad \mathbf{A}_{02}=\left[\begin{array}{rr}-3 & 0 \\ 5 & 2\end{array}\right], \quad \mathbf{A}_{12}=\left[\begin{array}{rr}1 & 0 \\ -5 & -5\end{array}\right]$

Let also assume that the delay is the same in the two modes i.e. $\tau_{1}=\tau_{2}$ $=1 s$ and the initial conditions are $\psi(t)_{-\tau \leq 0}=[1,1]^{\prime}$. Finally let the generator matrix $\Pi$ of the stochastic process $\mathbf{r}(t)$ be:

$$
\Pi=\left[\begin{array}{rr}
-1 & 1 \\
2 & -2
\end{array}\right]
$$

A program using Matlab has been developed to simulate the evolution of this dynamic system. The simulation results are shown in figure 2, where the curves of the variables $x_{1}(t), x_{2}(t)$ and $r(t)$ versus time are plotted. According to these curves, we conclude that the system is stochastically unstable.

The solution of the coupled Lyapunov equation given by (16) of nondelayed LSMJP is:

$$
P_{1}=\left[\begin{array}{cc}
1 & 0 \\
0 & 0.5263
\end{array}\right]_{2}=\left[\begin{array}{cc}
0.5 & 0 \\
0 & 0.3158
\end{array}\right]
$$

These matrices are symmetric and positive-definite, which means that the LSMJP is stable. Therefore, we see that the stability of the LSMJP does not imply the stability of the LTDSMJS. The reverse is true. 

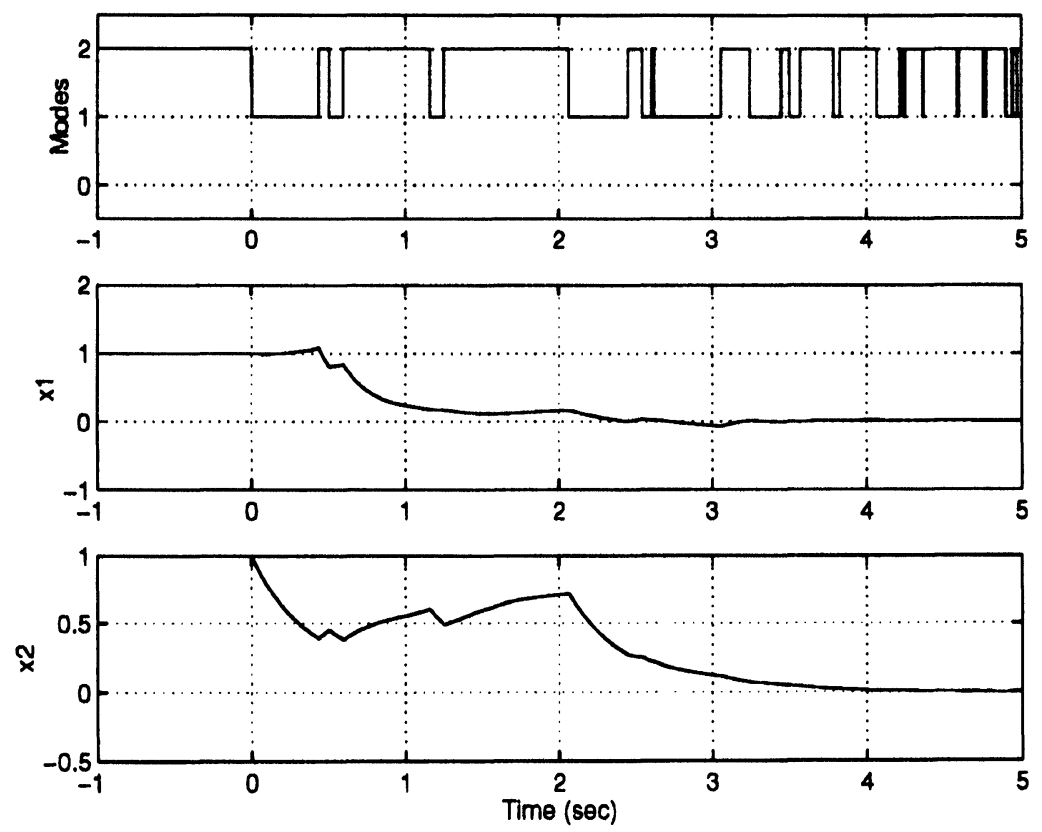

FIGURE 2 Evolution modes and states versus time.

\section{CONCLUSION AND FUTURE DIRECTIONS}

This paper deals with the class of linear time-delay systems with Markovian jumping parameters. A delay-independent necessary condition and sufficient conditions for stochastic stability of this class of systems have been established. To overcome the computation of the Riccati equations a delay-independent sufficient condition was proposed. Some examples have be given to show the proposed results. The delay-dependent mode case remains an open question.

\section{References}

[1] I.I. Kats, and N.N. Krasovskii. (1960) On the Stability of Systems with Random Attributes. J. Appl. Math. Mech. 24, 1225-1246.

[2] D.D. Sworder. (1969) Feedback Control of a class of Linear Systems with Jump Parameters. IEEE Trans. Automat. Control, 14, 9-14.

[3] M. Mariton, and P. Bertrand. (1985) Output Feedback for a Class of Linear Systems with Stochastic Jump Parameters. IEEE Trans. Automat. Control AC-30(9), 898-900.

[4] H.J. Chizeck, A.S. Willsky, and D. Castanon. (1986) Discrete-time Markovian Jump Linear Quadratic Control. Internat. J. Control 43(1), 219-234. 
[5] Y. Ji, and H.J. Chizeck. (1988) Controllability, Observability and Discrete-time Markovian Jump Linear Quadratic Control. Internat. J. Control 48(2) 481-498.

[6] Y. Ji, and H.J. Chizeck. (1990) Controllability, Stabilizability, and Continuous-time Markovian Jump Linear Quadratic Control. IEEE Trans. Automat. Control 35(7), 777788.

[7] H.J. Kushner. 1967. Stochastic Stability and Control, New York: Academic.

[8] M. Mariton. 1990. Jump Linear Systems in Automatic Control, New York and Basel.

[9] X. Feng, K.A. Loparo, Y. Ji, and H.J. Chizeck. (1992) Stochastic Stability Properties of Jump Linear Systems. IEEE Trans. Automat. Control 37(1), 38-53.

[10] E.K. Boukas. (1993) Control of Systems with Controlled Jump Markov Disturbances. Control-Theory and Adv. Tech. 9(2), 577-595.

[11] A. Thowsen. (1981) Stability of Time-delay Systems with Sampled Feedback. Internat. J. Control 34, 835-840.

[12] E. Cheres, S. Gutman, and Z.J. (1989) Palmor. Stabilization of Uncertain Dynamic System Including State Delay. IEEE Trans. Automat. Control 34, 1199-1230.

[13] M. Ikeda, and T. Ashida. (1979) Stabilization of Linear System with Time-varying Delay. IEEE Trans. Automat. Control AC-24, 369-370.

[14] R.M. Lewis, and B.D.O. Anderson. (1980) Necessary and Sufficient Conditions for Delay-independent Stability of Linear Autonomous Systems. IEEE Trans. Automat. Control AC-25, 735-739.

[15] S.D. Brierley, J.N. Chiasson, and S.H. Zak. (1982) On Stability Independent of Delay for Linear Systems. IEEE Trans. Automat. Control AC-27, 252-254.

[16] T. Mori, and H. Kokame. (1989) Stability of $\dot{x}(t)=A x(t)+B x(t-\tau)$. IEEE Trans. Automat. Control 34(4), 460-462.

[17] V.B. Kolmanovskii, and V.R. Nosov. 1986 Stability of Function Differential Equations, London: Academic.

[18] J. Gregory, and Nazaroff. (1973) Stability and Stabilization of Linear Differential Delay Systems. IEEE Trans. Automat. Control 317-318.

[19] H.K. Khalil. 1992 Nonlinear Systems, Macmillan Publishing Company, New York.

[20] E. Kreindler, and A. Jameson. (1972) Conditions for Nonnegativeness of Partitioned Matrices. IEEE Trans. Automat. Control AC-17, 147-148.

[21] H. Abou-Kandil, G. Freiling, and G. Jank. (1994) Solution and Asymptotic Behavior of Coupled Riccati Equations in Jump Linear Systems. IEEE Trans. Automat. Control 39(8), 1631-1636. 


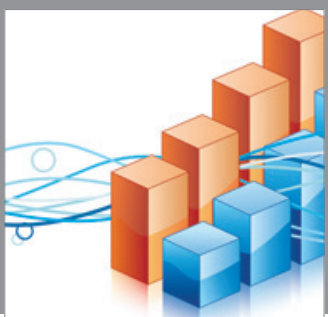

Advances in

Operations Research

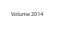

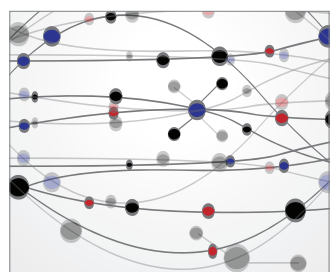

\section{The Scientific} World Journal
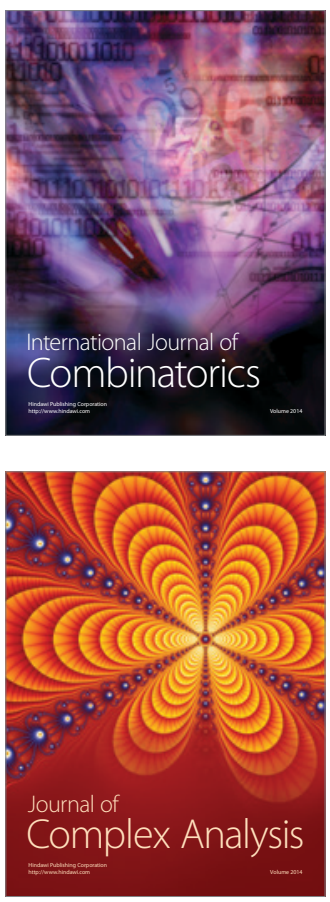

International Journal of

Mathematics and

Mathematical

Sciences
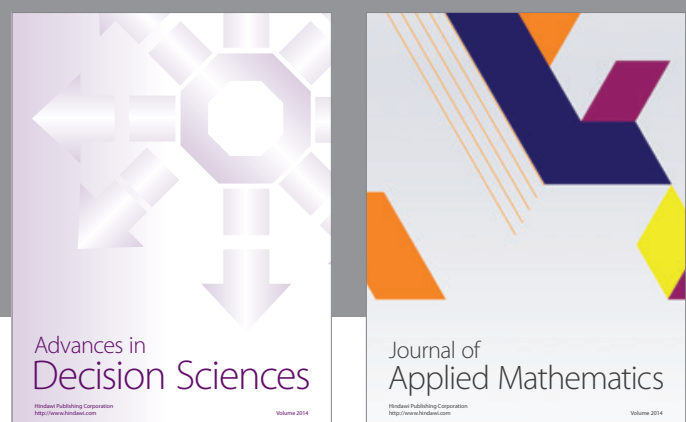

Journal of

Applied Mathematics
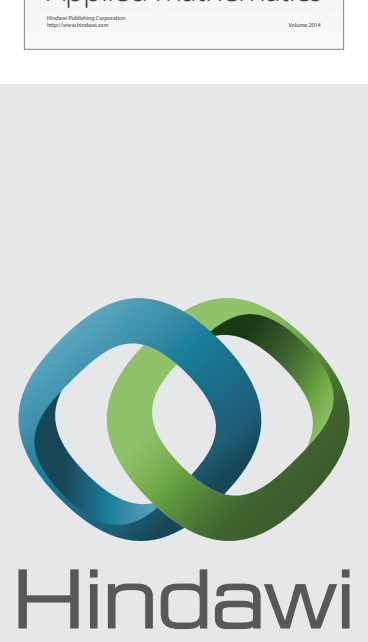

Submit your manuscripts at http://www.hindawi.com
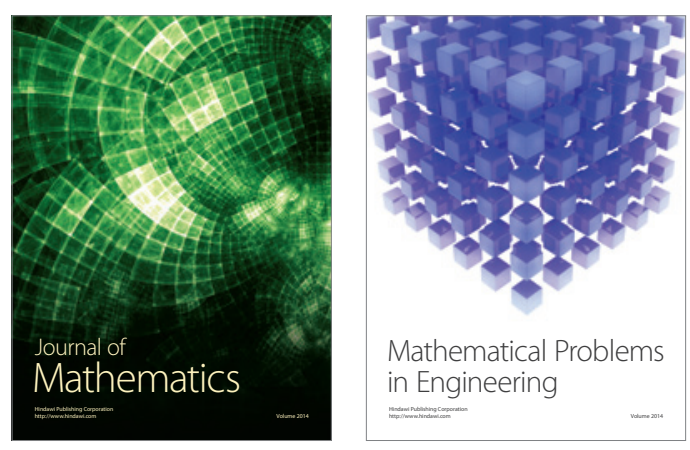

Mathematical Problems in Engineering
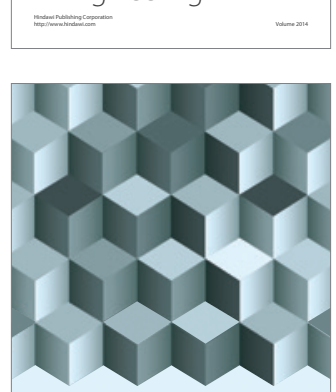

Journal of

Function Spaces
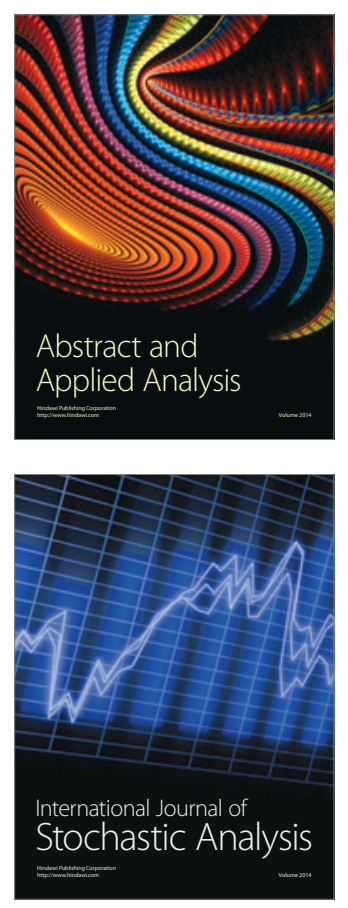

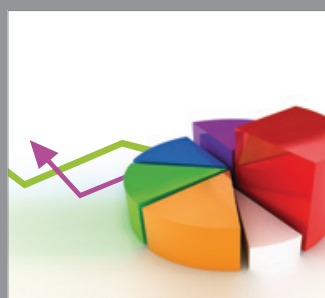

ournal of

Probability and Statistics

Promensencen
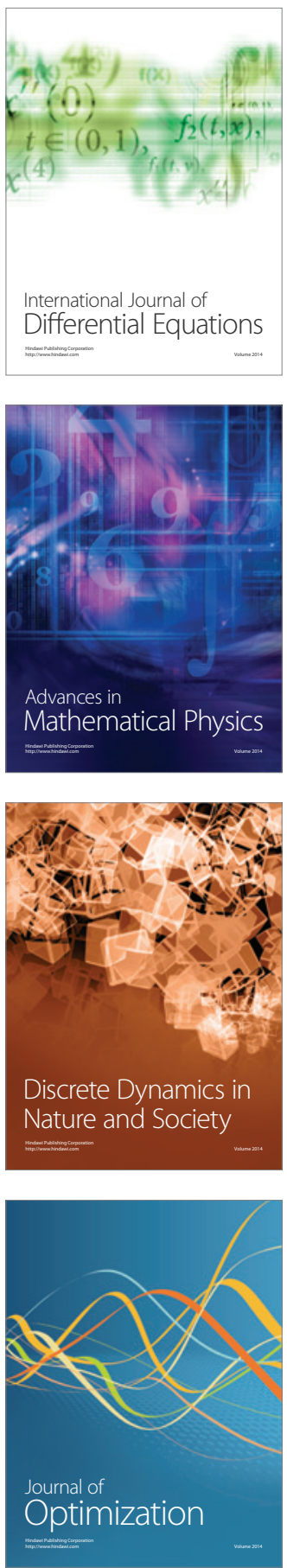